\title{
The Brauer Group of a Rational Surface
}

\section{J. S. Milne (Ann Arbor, Mich.)}

Let $k$ be a finite field of characteristic $p$ and let $X$ be an algebraic surface which is projective and smooth over $k$ and which is geometrically connected. Then, motivated by the relation between Brauer groups and Tate-Safarevič groups, Tate and Artin have conjectured [6]:

(a) the Brauer group, $\operatorname{Br}(X)$, of $X$ is finite;

(b) there is a canonical non-degenerate skew-symmetric form on $\operatorname{Br}(X)$;

(c) $P_{2}\left(X, q^{-s}\right) \sim \frac{[B r(X)]\left|\operatorname{det}\left(D_{i} \cdot D_{j}\right)\right|\left(1-q^{1-s}\right)^{\rho(X)}}{q^{\alpha(X)}\left[N S(X)_{\text {tors }}\right]^{2}} \quad$ as $s \rightarrow 1$,

where $[S]$ denotes the order of a set $S, q=[k], \alpha(X)=\chi\left(X, O_{X}\right)-1+$ $\operatorname{dim}(\operatorname{Pic} \operatorname{Var}(X)), \rho(X)$ is the rank of the Néron-Severi group $N S(X)$ of $X,\left(D_{i}\right)_{1 \leqq i \leqq \rho}$ is a basis for $N S(X)$ modulo torsion, and $P_{2}(X, T)$ is the characteristic polynomial of the endomorphism of $H_{l}^{2}\left(\bar{X}_{\mathrm{et}}\right)$ induced by the Frobenius endomorphism of $X$.

It has been proved [6] that (a) implies (b) and (c) for the components of $\operatorname{Br}(X)$ prime to $p$, and when $X$ is a product of curves the conjectures have been proved in their entirety [4]. Nevertheless, it may be of interest that for the simplest surfaces, viz. the rational surfaces, the conjectures are an almost trivial consequence of known facts.

Thus, let $X$ be a rational surface over $k$ of the above type, let $\vec{k}$ be the algebraic closure of $k$, and let $k^{\prime}$ be a finite extension of $k$ such that $N S\left(X^{\prime}\right)=N S(\bar{X})$ where $X^{\prime}=X \otimes_{k} k^{\prime}$ and $\bar{X}=X \otimes_{k} \bar{k}$. Write $\Gamma$ and $\Gamma^{\prime}$ for the Galois groups of $\vec{k}$ over $k$ and $k^{\prime}$ respectively and write $\Gamma^{\prime \prime}=\Gamma / \Gamma^{\prime}$.

$N S(\bar{X})$ is torsion-free and the pairing $N S(\bar{X}) \times N S(\bar{X}) \rightarrow \mathrm{Z}$ defined by the intersection product has discriminant \pm 1 . Indeed, both these statements are true for $\mathbf{P}_{k}^{2}$ and their validity is obviously preserved by dilations.

The Brauer group of $X$ is isomorphic to $H^{1}(\Gamma, N S(\bar{X}))$. This remark is due to Artin and may be proved as follows. The Hochschild-Serre spectral sequence for $\vec{X}_{\mathrm{et}} / X_{\mathrm{et}}$ applied to the sheaf $\mathbf{G}_{m}$ gives an exact sequence

$$
0 \rightarrow H^{1}(\Gamma, N S(\bar{X})) \rightarrow H^{2}\left(X, \mathbf{G}_{m}\right) \rightarrow H^{2}\left(\bar{X}, \mathbf{G}_{m}\right) .
$$


$H^{2}\left(\bar{X}, \mathbf{G}_{m}\right)=\operatorname{Br}(\bar{X})=0$ because $\operatorname{Br}(\bar{X})$ is birationally invariant [1] and $\bar{X}$ is birationally equivalent to $\mathbf{P}_{k}^{1} \times \mathbf{P}_{k}^{1}$. If $f: \mathbf{P}_{k}^{1} \times \mathbf{P}_{k}^{1} \rightarrow \mathbf{P}_{k}^{1}$ denotes a projection onto one of the factors then $R^{0} f_{*} \mathbf{G}_{m}=\mathbf{G}_{m}, R^{1} f_{*} \mathbf{G}_{m}=\mathbf{Z}$, and $R^{s} f_{*} \mathbf{G}_{m}=0$ for $s>1$. Since $H^{r}\left(\mathbf{P}_{k}^{1}, \mathbf{G}_{m}\right)=0$ for $r>1$ and $H^{1}\left(\mathbf{P}_{k}^{1}, \mathbf{Z}\right)=0$, the Leray spectral sequence for $f$ shows that $H^{2}\left(\mathbf{P}_{k}^{1} \times \mathbf{P}_{k}^{1}, \mathbf{G}_{m}\right)=0$. Hence $H^{\mathbf{1}}(\Gamma, N S(\bar{X})) \approx B r(X)$.

There is an exact sequence

$$
0 \rightarrow H^{1}\left(\Gamma^{\prime \prime}, N S\left(X^{\prime}\right)\right) \rightarrow H^{1}(\Gamma, N S(\bar{X})) \rightarrow H^{1}\left(\Gamma^{\prime}, N S(\bar{X})\right) .
$$

$\Gamma^{\prime}$ acts trivially on $N S(\bar{X})$, and so

$$
H^{1}\left(\Gamma^{\prime}, N S(\bar{X})\right)=\text { Conts } \operatorname{Hom}\left(\Gamma^{\prime}, N S(\bar{X})\right),
$$

which is zero because $N S(\bar{X})$ has no finite subgroups. Hence

$$
B r(X) \approx H^{1}(\Gamma, N S(\bar{X})) \approx H^{1}\left(\Gamma^{\prime \prime}, N S\left(X^{\prime}\right)\right) .
$$

This last group is finite because $\Gamma^{\prime \prime}$ is finite and $N S\left(X^{\prime}\right)$ is finitely generated. This proves (a).

$\mathbf{Z}$, regarded as a $\Gamma^{\prime \prime}$ module with trivial action, is a class module for $\Gamma^{\prime \prime}$ in the sense of $[2$, p. 94]. Since the intersection product induces a natural isomorphism $N S(\bar{X}) \approx \operatorname{Hom}(N S(\bar{X}), Z)$, [2, IV Thm. 14] shows that the cup-product pairing

$$
H^{1}\left(\Gamma^{\prime \prime}, N S(\bar{X})\right) \times H^{1}\left(\Gamma^{\prime \prime}, N S(\bar{X})\right) \rightarrow H^{2}\left(\Gamma^{\prime \prime}, \mathbf{Z}\right) \approx \mathbf{Z} / n \mathbf{Z} \quad\left(n=\left[\Gamma^{\prime \prime}\right]\right)
$$

is non-degenerate. This pairing agrees with the pairing on $\operatorname{Br}(X)$ (non $p$ ) defined in [6]. The general properties of cup-products show that the pairing is skew-symmetric but (pace [6, p. 19]) it need not be alternating and so the order of $\operatorname{Br}(X)$ may be twice a square. For examples where $[\operatorname{Br}(X)]=2$, see $[3,3.28]$. This completes the proof of $(b)$.

For (c), consider the commutative diagram:

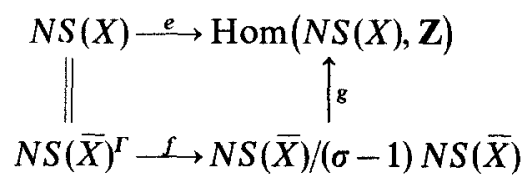

where $\sigma$ is the canonical topological generator of $\Gamma, f$ is induced by the identity map of $N S(\bar{X})$, and $e$ and $g$ are both induced by the intersection product. We will say that a homomorphism $h$ of $\mathbf{Z}$-modules is a quasi-isomorphism if both $\operatorname{ker}(h)$ and coker $(h)$ are finite, and in that case we write $z(h)=\frac{[\operatorname{coker}(h)]}{[\operatorname{ker}(h)]}$. Lemmas analogous to those on pp.19, 20 of [6] hold for this definition of $z$. In particular, $z(e)=\left|\operatorname{det}\left(D_{i} \cdot D_{j}\right)\right|$ 
where $\left(D_{i}\right)$ is a basis for $N S(X)$. (Notice that, unlike the corresponding determinant for $N S(\bar{X})$, this need not be 1 . For example, if $X$ is a nondegenerate del Pezzo surface whose degree $d$ is square-free, then $\left.\left|\operatorname{det}\left(D_{i} \cdot D_{j}\right)\right|=\left(\omega_{X} \cdot \omega_{X}\right)=d.\right)$

Consider the pairing

$$
N S(\bar{X}) \times N S(X) \rightarrow Z
$$

defined by the intersection product. Suppose $D \in N S(\bar{X})$ is such that $N D=\sum_{i=0}^{n-1} \sigma^{i} D=0$ (where $n=\left[\Gamma^{\prime \prime}\right]$, so $\left.\Gamma^{\prime \prime}=\left\{1, \bar{\sigma}, \ldots, \bar{\sigma}^{n-1}\right\}\right)$. Then, for any $E \in N S(X), n(D \cdot E)=\sum_{i=0}^{n-1}\left(D \cdot \sigma^{-i} E\right)=(N D \cdot E)=0$.

Hence $(D \cdot E)=0$. Conversely, if $(D \cdot E)=0$ for all $E \in N S(X)$ then $(N D \cdot E)=n(D \cdot E)=0$ for all $E$, and since $N D \in N S(X)$, this implies that $N D=0$. This shows that the kernel of $g$ is $\operatorname{ker}(N: N S(\bar{X}) \rightarrow N S(\bar{X}))$ / $(\sigma-1) N S(\bar{X})=H^{1}\left(\Gamma^{\prime \prime}, N S(\bar{X})\right)$. Since $g$ is obviously surjective, we find that $z(g)=[B r(X)]^{-1}$.

The étale cohomology sequence of

$$
0 \rightarrow \mu_{l^{n}} \rightarrow \mathbf{G}_{m} \stackrel{l^{n}}{\longrightarrow} \mathbf{G}_{m} \rightarrow 0 \quad(l \neq p)
$$

gives an isomorphism $N S(\bar{X}) / l^{n} N S(\bar{X}) \approx H^{2}\left(\bar{X}, \mu_{l^{n}}\right)$. Hence

$$
N S(\bar{X}) \otimes \mathbf{Z}_{l} \approx \lim _{\longleftarrow} H^{2}\left(X, \mu_{l^{n}}\right),
$$

and $N S(\bar{X}) \otimes \mathbf{Q}_{l} \approx H_{l}^{2}(\bar{X})(1)$ in the notation of [5]. Thus [5, p. 101] if $\sigma_{2}$ is the automorphism of $N S(\bar{X}) \otimes Q_{l}$ induced by $\sigma$ then $\operatorname{det}\left(1-\sigma_{2} T\right)=$ $P_{2}\left(X, q^{-1} T\right)$ (see also [7]). $g$ and $e$ both being quasi-isomorphisms imply that $f$ is a quasi-isomorphism. Thus, by the analogue of $[6, z .4]$, if $\theta$ is the map $\sigma-1: N S(\bar{X}) \rightarrow N S(\bar{X})$, then $\operatorname{det}(T-\theta \otimes 1)=T^{\rho} R(T)$ where $\rho=\operatorname{rank}(N S(X))$. Also, $z(f)=R(0)=\Pi\left(1-\frac{\alpha_{i}}{q}\right)$ where the $\alpha_{i}$ are the roots of $P_{2}(X, T)$ which are not equal to $q$. Now the equality $z(f) z(g)=z(e)$ shows that

$$
P_{2}\left(X, q^{-s}\right) \sim[B r(X)]\left|\operatorname{det}\left(D_{i} \cdot D_{j}\right)\right|\left(1-q^{1-s}\right)^{\rho(X)} \quad \text { as } s \rightarrow 1 .
$$

This implies (c) because in this case $\alpha(X)=1-1+0=0$.

Example. Let $k$ contain the cube roots of 1 and have characteristic $\neq 3$, and let $a$ be an element of $k$ which is not a cube in $k$. Then

$$
X: Z_{0}^{3}+Z_{1}^{3}+Z_{2}^{3}=a Z_{3}^{3}
$$

is a rational surface which over $k^{\prime}=k(3 \sqrt{a})$, becomes isomorphic to $\mathbf{P}_{k}^{2}$, with 6 points blown up. Moreover, $N S(X)$ has rank 1 . It follows 
(using that $N S(\bar{X})$ has rank 7 and that $\Gamma^{\prime \prime} \approx \mathbf{Z} / 3 \mathbf{Z}$ has only one nontrivial representation over $\mathbf{Q}$ ) that

$$
P_{2}(X, T)=(1-q T)(1-\rho q T)^{3}\left(1-\rho^{2} q T\right)^{3}
$$

where $\rho$ is a primitive cube root of 1 . Hence

$$
P_{2}\left(X, q^{-s}\right) \sim 27\left(1-q^{1-s}\right) \quad \text { as } s \rightarrow 1 .
$$

By Noether's formula $\left(\omega_{X} \cdot \omega_{X}\right)+\operatorname{rank}(N S(\bar{X}))=10$, and so $\left(\omega_{X} \cdot \omega_{X}\right)=3$. It follows that $\omega_{X}$ generates $N S(X)$ and that $[\operatorname{Br}(X)]=9$. Because of the self-duality of $B r(X)$, this implies that

$$
\operatorname{Br}(X) \approx \mathbf{Z} / \mathbf{3 Z} \oplus \mathbf{Z} / \mathbf{3 Z} \text {. }
$$

\section{References}

1. Grothendieck, A.: Le groupe de Brauer III. Dix Exposes sur la Cohomologie des Schemas, p. 88-188. Amsterdam: North-Holland; Paris: Masson 1968.

2. Lang, S.: Rapport sur la cohomologie des groupes. New York: Benjamin 1966.

3. Manin, Ju.: Rational surfaces over petfect fields I. Inst. Hautes Etudes Sci. Publ. Math. 30, 55-97 (1966).

4. Milne, J.: The Tate-Šafarevič group of a constant abelian variety. Inventions math. 6, 91-105 (1968).

5. Tate, J.: Algebraic cycles and poles of zeta functions. Proc. Purdue Univ. Conf. 1963, p. 93-110. New York: Harper \& Row 1965.

6. - On the conjectures of Birch and Swinnerton-Dyer and a geometric analogue. Séminaire Bourbaki 1965/66, Exposé 306. New York: Benjamin 1966.

7. Weil, A.: Abstract versus classical algebraic geometry, Proc. Internat. Congress Math. (Amsterdam 1954), vol. 3, p. 550-558. Amsterdam: North-Holland 1956.

J.S. Milne

The University of Michigan

Dept. of Mathematics

Ann Arbor, Michigan 48105, USA

(Received September 28, 1970) 